\title{
Semantic Analysis Does Not Occur in the Absence of Awareness Induced by Interocular Suppression
}

\author{
Min-Suk Kang, ${ }^{1}$ Randolph Blake, ${ }^{1,2}$ and Geoffrey F. Woodman ${ }^{1}$ \\ ${ }^{1}$ Department of Psychology, Vanderbilt Vision Research Center, Center for Cognitive and Integrative Cognitive Neuroscience, Vanderbilt University, \\ Nashville, Tennessee 37240-7817 and '2Department of Brain and Cognitive Sciences, Seoul National University, Seoul 151-742, Korea
}

It has been intensely debated whether visual stimuli are processed to the point of semantic analysis in the absence of awareness. In the present study, we measured the extent to which the meaning of a stimulus was registered using the N400 component of human eventrelated potentials (ERPs), a highly sensitive index of the semantic mismatch between a stimulus and the context in which it is presented. Observers judged the semantic relatedness of a context and target word while ERPs were recorded under continuous flash suppression (Experiments 1 and 2) and binocular rivalry (Experiment 3). Finally, we parametrically manipulated the visibility of the target word by increasing the contrast between the target word and the suppressive stimulus presented to the other eye (Experiment 4). We found that the amplitude of the N400 was attenuated with increasing suppression depth and was absent whenever the observers could not discriminate the meaning of suppressed words. We discuss these findings in the context of single-process models of consciousness, which can account for a large body of empirical evidence obtained from visual masking, attentional manipulations, and, now, interocular suppression paradigms.

\section{Introduction}

Interocular suppression represents a potentially powerful means for learning what aspects of visual processing transpire even in the absence of awareness (Kim and Blake, 2005; Lin and He, 2009) and for identifying brain areas involved in those visual processes (Haynes and Rees, 2005; Wunderlich et al., 2005; Tong et al., 2006; Lee et al., 2007; Sterzer et al., 2008). In this paper, we focus on the unresolved debate about whether a visual stimulus blocked from awareness by interocular suppression still undergoes semantic analysis, because previous work provides conflicting answers to this question.

Results from several psychophysical studies indicate that the meaning of a stimulus is not registered during interocular suppression, where "meaning" can refer to the semantics of written words (Zimba and Blake, 1983; Blake, 1988) or to the identity of everyday objects (Cave et al., 1998; Moradi et al., 2005). However, other findings point to a different conclusion. For example, it has been reported that stimuli blocked from awareness by interocular

Received April 4, 2011; revised Aug. 2, 2011; accepted Aug. 4, 2011.

Author contributions: M.-S.K., R.B., and G.F.W. designed research; M.-S.K. performed research; M.-S.K. analyzed data; M.-S.K., R.B., and G.F.W. wrote the paper.

This work was supported by R01 EY019882 from the National Eye Institute (NEI) of NIH and BCS-0957072 from the National Science Foundation (G.F.W.), by R01 EY13358 from the NEl and by the World Class University program through the National Research Foundation of Korea funded by the Ministry of Education, Science and Technology under the auspices of R32-10142 (R.B.), and by NIH Core Grant P30-EY008126 (Vanderbilt Vision Research Center). We thank Hyeon-Ae Jeon, Timothy McNamara, and Edward Vogel for contributing to the list of related words. Timothy McNamara, Bruce McCandless, Gordon Logan, and Sheng He provided crucial input during this project and the preparation of the manuscript.

The authors declare no financial conflict of interest.

Correspondence should be addressed to Min-Suk Kang, Vanderbilt University, Wilson Hall, PMB 407817, 2301 Vanderbilt Place, Nashville, TN 37240-7817. E-mail: m.suk.kang@gmail.com.

DOI:10.1523/JNEUROSCI.1691-11.2011

Copyright $\odot 2011$ the authors $\quad 0270-6474 / 11 / 3113535-11 \$ 15.00 / 0$ suppression nonetheless can guide spatial attention (Jiang et al., 2006) and facilitate responses to meaningfully congruent stimuli presented immediately after these invisible inputs (Almeida et al., 2008). Moreover, target words presented under binocular rivalry suppression become visible earlier if they are semantically related to clearly visible context words compared with trials in which the suppressed words are semantically unrelated to the context words (Costello et al., 2009).

In the present study, we used a sensitive electrophysiological index of semantic processing, the N400 component, to clarify the extent to which visually presented words are processed in the absence of awareness. The N400 is an event-related potential (ERP) waveform that measures the degree to which two stimuli are semantically congruent. For example, when a clearly visible context word and target word are presented in sequence, the target word produces a more negative-going potential beginning 300-400 ms poststimulus when that target word is semantically unrelated to the context word compared with when the context and target words are semantically related (Kutas and Hillyard, 1980; Lau et al., 2008; Kutas and Federmeier, 2011). The advantage of using this ERP component is that we can directly measure brain activity during this specific cognitive operation even in the absence of awareness of the meaningful stimuli that ordinarily engage that operation (Luck et al., 1996; Vogel et al., 2005).

\section{Materials and Methods}

Participants. Thirty-eight adults with normal color vision, acuity, and neurological history volunteered and were compensated $\$ 10$ per hour for their participation. Six participated in Experiment 1 (three females), eight participated in Experiment 2 (six females), eight in Experiment 3 (four females), and 16 in Experiment 4 (11 females). All volunteers provided informed consent before their participation in the study, which 

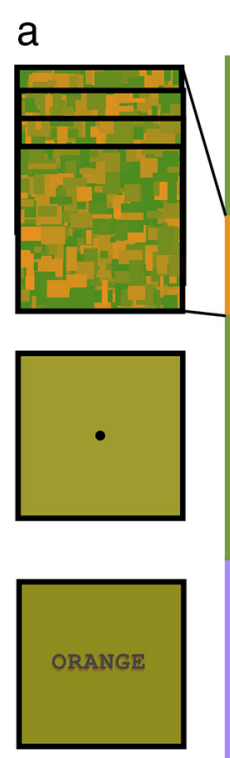

Left Eye
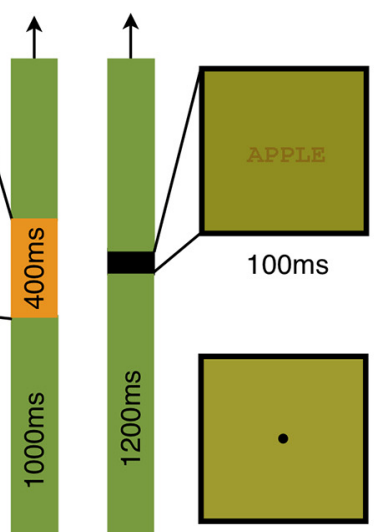

b

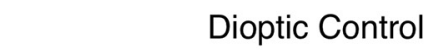

$10^{-10}$

$\mu \mathrm{V}$
C
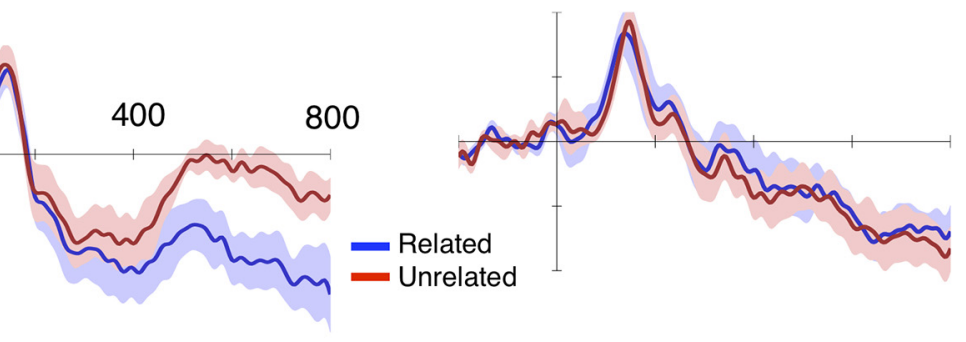

Suppression

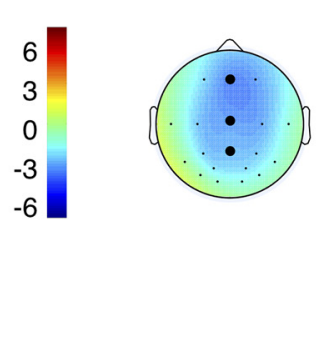

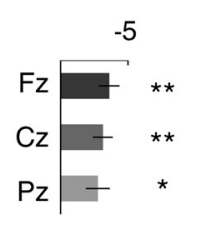
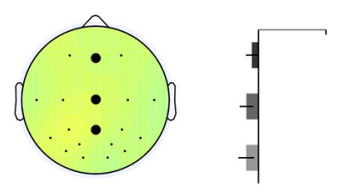

Figure 1. Stimuli and results of Experiment 1. $a$, Illustration of stimulus sequence of Experiment 1 (related-pair type of a suppression trial). Context word (ORANGE) is presented to the two eyes for $1 \mathrm{~s}$ and the first Mondrian frame is presented to one eye after the $1 \mathrm{~s}$ fixation. Four Mondrian frames are presented in sequence, one every $100 \mathrm{~ms}$. The target word (APPLE) is presented the other eye for $100 \mathrm{~ms}$ with the third Mondrian frame. $\boldsymbol{b}, \boldsymbol{c}$, Results of Experiment $1(\mathrm{~N}=6)$. ERP waveforms and the N400 amplitude obtained from dioptic-control $(\boldsymbol{b})$ and suppression $(\boldsymbol{c})$ trials. ERP waveforms elicited by the related targets are in blue and by the unrelated targets are in red. The shaded regions of matching color indicate the $\pm 1 \mathrm{SE}$ between subjects. $\boldsymbol{b}, \boldsymbol{c}$, Bottom, N400 amplitude measured for all electrodes represented by topographical map and the N400 amplitude of the three key electrodes (Fz, Cz, and Pz) are plotted to the right of the map. The N400 amplitude was obtained from the difference waves (unrelated pairs - related pairs) during the temporal window of $350-500 \mathrm{~ms}$ after the onset of the target word. The N400 amplitudes of the topographical map are color coded ( $\boldsymbol{b}$, color bar) and $t$ test significance is indicated with asterisks $\left({ }^{*} p<0.05,{ }^{* *} p<0.01\right)$ for the N400 amplitudes of the three key electrodes.

was approved by Vanderbilt University's Institutional Review Board. We excluded observers whose semantic judgment accuracy was $>60 \%$ correct under conditions of suppression because of a concern that suppression might be incomplete using the range of luminance values available with our monitor.

Apparatus. Observers were seated $114 \mathrm{~cm}$ from a computer screen and responded using a gamepad (Logitech Precision). All stimuli were presented on a light green background $\left(x=0.41, y=0.51 ; 47.5 \mathrm{~cd} / \mathrm{m}^{2}\right)$ using the Psychophysics Toolbox-3 (Brainard, 1997; Pelli, 1997) in conjunction with Matlab (Mathworks). Two rival stimuli were presented to the two eyes using the anaglyph method (i.e., red and green glasses).

Electrophysiological recording and analysis. We recorded the EEG from tin electrodes embedded in an elastic cap (Electro-Cap International). The caps contained a subset of the International 10/20 System sites (Fz, $\mathrm{Cz}, \mathrm{Pz}, \mathrm{F} 3, \mathrm{~F} 4, \mathrm{C} 3, \mathrm{C} 4, \mathrm{P} 3, \mathrm{P} 4, \mathrm{PO} 3, \mathrm{PO} 4, \mathrm{~T} 3, \mathrm{~T} 4, \mathrm{~T} 5, \mathrm{~T} 6, \mathrm{O} 1$, and O2) in addition to two nonstandard sites (OL, placed halfway between $\mathrm{O} 1$ and T5; OR, placed halfway between $\mathrm{O} 2$ and T6). These electrodes were referenced online to the right mastoid, and re-referenced offline to the average of the right and left mastoid (Nunez and Srinivasan, 2006). We recorded the horizontal EOG from two electrodes placed $\sim 1 \mathrm{~cm}$ from the external canthus of each eye and an electrode placed $\sim 3 \mathrm{~cm}$ below the left eye, referenced to the right mastoid, measured vertical EOG. Signals were amplified using an SA Instrumentation amplifier with a gain of 20,000 and a bandpass of $0.01-100 \mathrm{~Hz}$. The amplified signals were digitized by a PC-compatible computer at a rate of $250 \mathrm{~Hz}$ and averaged offline. We rejected individual trials with eye movements, blinks, muscle noise, or amplifier saturation before averaging. The ERP waveforms were timelocked to the onset of the target words and baseline corrected to the interval -200 to $0 \mathrm{~ms}$ before target-word onset. Waveforms were lowpass filtered (two-way least-squares finite impulse response filtering with 0 and $35 \mathrm{~Hz}$ for low and high ends of the frequency band, respectively) for presentation in the figures only; the analyses were performed on the unfiltered mean voltages.

Stimuli and procedure of Experiment 1. To create interocular suppression, we generated Mondrian patches that filled a square frame (Tsuchiya and Koch, 2005). Within each square Mondrian frame $\left(4.7^{\circ} \times 4.7^{\circ}\right), 200$ rectangular patches of different luminance $\left(3-61 \mathrm{~cd} / \mathrm{m}^{2}\right)$ and size $(0.1-$ $0.8^{\circ}$ edge lengths) were randomly drawn over different positions. The contrast of the entire Mondrian was calculated by computing the difference between the maximum and the minimum luminance levels of the rectangular patches divided by the maximum luminance range of the monitor. The random luminance levels of the rectangular patches that composed each Mondrian were constrained such that the mean luminance level of each Mondrian was approximately equal to the average background luminance level $\left(47.5 \mathrm{~cd} / \mathrm{m}^{2}\right)$. During each trial, four Mondrian frames were presented for $100 \mathrm{~ms}$ each in a contiguous temporal sequence. Target words were drawn using capital letters at 30\% contrast and context words were drawn at $100 \%$ contrast. The contrast of the words was calculated by the luminance difference between the background and the luminance of the words divided by the luminance of the background. Each character was presented in a monospace regular Courier font and was $\sim 0.37^{\circ} \times 0.37^{\circ}$. On suppression trials, the target word and Mondrian patches were presented to different eyes; for the diopticcontrol trials, the same target words were also embedded within the third Mondrain patch so that the target word was presented to both eyes. A black frame $\left(4.7^{\circ} \times 4.7^{\circ}\right.$; line width, $\left.0.24^{\circ}\right)$ was presented dioptically to promote binocular fusion.

Our word stimuli consisted of 240 related word pairs drawn from previous N400 studies (Luck et al., 1996; Vogel et al., 1998). Four sets of related pairs of words were prepared with 60 related pairs of words in each set. For each observer, two sets were assigned to the suppression trials and the other two sets were used for the dioptic-control trials. Within each trial type, one set was used for related pairs and the other was used to create unrelated pairs by swapping target words (Vogel et al., 1998). To equate stimulus energy across observers, the stimuli were counterbalanced across related versus unrelated trial types.

Figure $1 a$ illustrates the stimulus sequence on each trial in Experiment 1. Each trial began with the presentation of a fixation point for $900 \mathrm{~ms}$ followed by the presentation of the context word to both eyes for $1 \mathrm{~s}$. Following another $1 \mathrm{~s}$ presentation of the fixation point, the Mondrian 


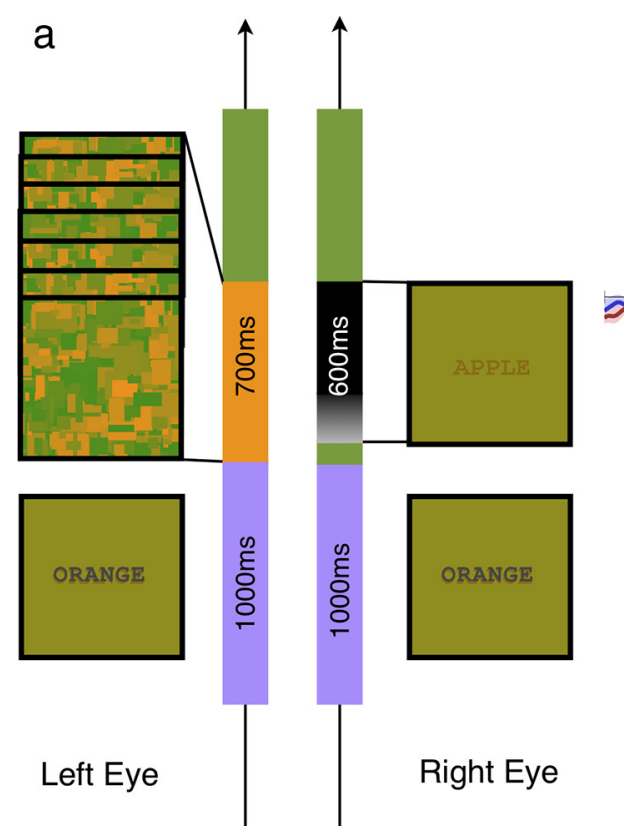

b

C

Dioptic Control

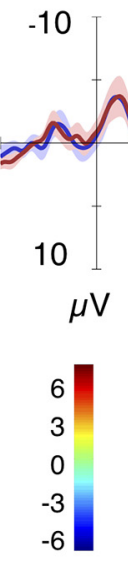

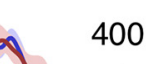

00
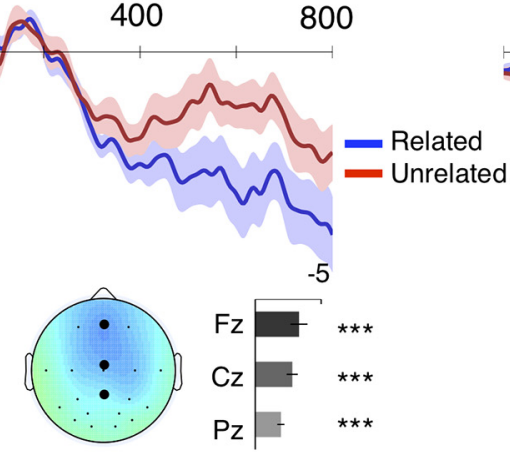

Suppression

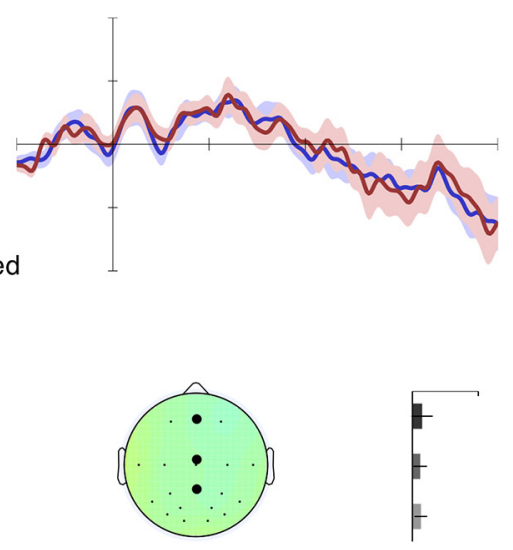

Figure 2. Stimuli and results of Experiment 2. $\boldsymbol{a}$, Illustration of stimulus sequence of Experiment 2 (related-pair type of a suppression trial). Context word (ORANGE) is presented to both eyes for $1 \mathrm{~s}$ and the first Mondrian frame is presented to one eye immediately. Seven Mondrian frames are presented in sequence, one every $100 \mathrm{~ms}$. The target word (APPLE) is presented the other eye after a $100 \mathrm{~ms}$ blank interval. The contrast of the target word was gradually increased for the first $300 \mathrm{~ms}$ and remained the same for another $300 \mathrm{~ms} . \boldsymbol{b}, \boldsymbol{c}$, Results of Experiment 2 ( $N=8$ ). ERP waveforms and the $\mathrm{N} 400$ amplitude obtained from dioptic-control (b) and suppression (c) trials. Details are as in Figure $1, b$ and $c$.

frames were presented to one eye for $400 \mathrm{~ms}$, changing their patterns every $100 \mathrm{~ms}$. A target word was presented for $100 \mathrm{~ms}$, beginning $200 \mathrm{~ms}$ after the onset of the Mondrian patches. On the dioptic-control trials, the target word was presented to both eyes; on the critical suppression trials, the target was presented only to the eye not shown the Mondrians. Observers were instructed to fixate for $500 \mathrm{~ms}$ after the offset of the Mondrian patches and then to report whether the pairs of words were semantically related or not by using their left or right index finger to press one of two buttons on the gamepad. The button mapping between left and right hands associated with each response (related/unrelated) was counterbalanced across observers. If observers were not aware of a given target word, they were required to make a response anyway by guessing. Responses were not speeded and accuracy was stressed.

Stimuli and procedure of Experiment 2. Experiment 2 was identical to Experiment 1, with the following exceptions. The target word onset and its duration were set to correspond to those used in a recent study by Costello et al. (2009). Specifically, the context word was presented for $1 \mathrm{~s}$ after a $900 \mathrm{~ms}$ fixation interval, and the Mondrian frames were presented immediately after the context word for $700 \mathrm{~ms}$, changing their patterns every $100 \mathrm{~ms}$. To reduce incomplete suppression associated with visual transients (Walker and Powell, 1979; Blake et al., 1990), the contrast of the target word was gradually increased $100 \mathrm{~ms}$ after the onset of the Mondrian frames, such that its contrast changed for the first $300 \mathrm{~ms}$ and then remained at its maximum contrast level for $300 \mathrm{~ms}$. The contrast of the target words was set individually for each observer. The contrast level was set at $33 \%$ at first and then reduced to $25 \%$ if observers could see at $33 \%$ contrast level (mean contrast level, $\sim 27 \%$ ). The contrast of the Mondrian frame was set to $100 \%$. Figure $2 a$ illustrates the stimulus sequence on each trial of Experiment 2.

Stimuli and procedure of Experiment 3. Red vertical gratings and green horizontal gratings of $30 \%$ contrast were presented to different eyes. The size of these grating stimuli was $2.9^{\circ} \times 2.9^{\circ}$ and their spatial frequency was $\sim 1$ cycle per degree. Before the experiment began, the contrast of context words was adjusted individually for each observer such that the observers could recognize the embedded context words when the horizontal grating was dominant but not when the vertical grating was dominant. This calibration was conducted using an iterative procedure in which the contrast of the context word was set to the highest contrast at which the observer could not report any of three consecutive words but one level below the contrast at which at least one word could be reported. Across observers, this resulted in the luminance of the context words ranging between $50 \%$ and $70 \%$ of the grating contrast (mean $=67 \%$ ). The trial type cues were the capital letters $\mathrm{V}$ or $\mathrm{H}$ drawn in the same font and size as the words and presented at fixation. All other aspects of stimuli were identical to Experiment 1.

Figure $3 a$ illustrates the stimulus sequence used in Experiment 3. Each trial began with a cue indicating whether the observers were supposed to trigger the presentation of context word when the vertical or horizontal grating was dominant. If the capital letter $\mathrm{V}$ was presented before the trial, observers had to wait until the vertical grating became dominant and pressed a button to trigger the context word onset. If the capital letter $\mathrm{H}$ was presented, observers had to wait until the horizontal grating became dominant to trigger the presentation of the context word. This instruction letter was presented for $1 \mathrm{~s}$ and the rival gratings were presented immediately thereafter. Once observers reported achieving the instructed dominance, the contrast of context words increased for $70 \mathrm{~ms}$ and then decreased for $70 \mathrm{~ms}$ with a sine wave profile with a period of 280 $\mathrm{ms}$. The rival gratings remained visible for $200 \mathrm{~ms}$ after the offset of the context word. After a $500 \mathrm{~ms}$ fixation period, a target word was presented for $1 \mathrm{~s}$. On each trial following an $\mathrm{H}$ cue (i.e., dominance trials), participants were asked to perform a semantic judgment task in which they pressed one button on the gamepad to indicate related and a different button to indicate unrelated. The button mappings were counterbalanced as in Experiment 1. On trials following the V cues (suppression trials), the participants were also instructed to indicate that they recognized the context word due to incomplete suppression by pressing a third button with their right-hand thumb. The set of word stimuli and all other procedures were identical to Experiment 1.

Stimuli and procedure of Experiment 4 . We compiled 384 semantically related pairs of words used in the previous studies (Besner et al., 1990; Luck et al., 1996; O'Seaghdha and Marin, 1997; Brown et al., 2006). We then divided them into eight sets. In each set, there were 48 semantically related pairs of words. The number of letters in each word ranged from three to seven. To precisely control for the stimulus energy associated with word length, the number of letters that comprised the prime and target words was closely matched across the sets such that the mean number of letters was 4.71 for the prime words and 4.73 for the target words. In addition, the frequency of word length was matched across the 

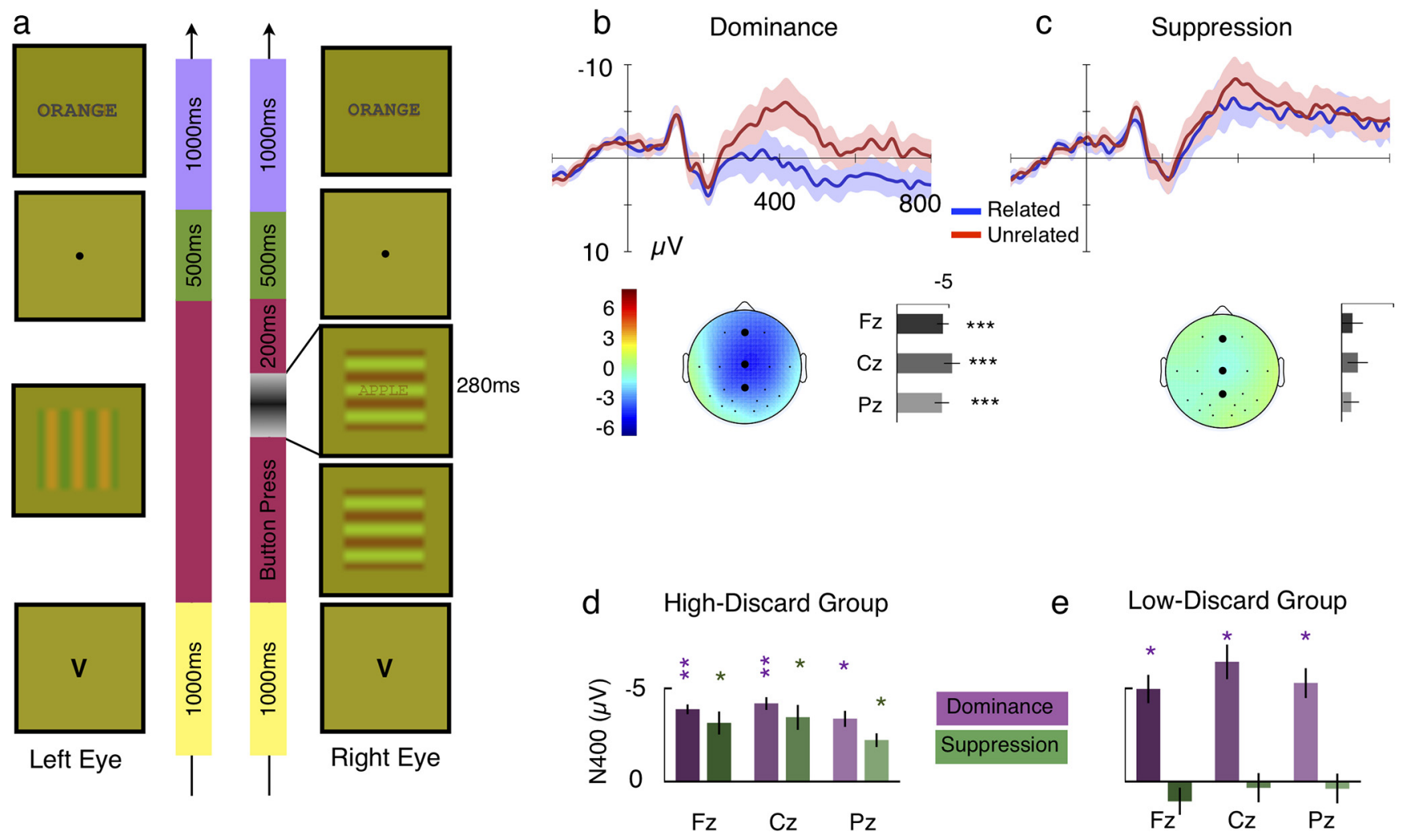

Figure 3. Stimuli and results of Experiment 3. $\boldsymbol{a}$, Illustration of stimulus sequence of Experiment 3. The vertical and the horizontal gratings were presented to different eyes. Once observers reported achieving the instructed dominance specified at the beginning of each trial by either $\mathrm{V}$ (vertical grating on suppression trials) or $\mathrm{H}$ (horizontal grating on dominance trials), the contrast of the context word increased and decreased gradually. The fixation was presented after the offset of the rival patterns for $500 \mathrm{~ms}$ and then the target word was presented for semantic judgment task. $\boldsymbol{b}-\boldsymbol{e}$, Results of Experiment $3(N=8) \cdot \boldsymbol{b}, \boldsymbol{c}$, ERP waveforms and the $\mathrm{N} 400$ amplitude obtained from dominance $(\boldsymbol{b})$ and suppression $(\boldsymbol{c})$ trials. Details are as in Figure $1, b$ and $c$. The $N 400$ amplitude at the three key electrodes ( $\mathrm{Fz}, \mathrm{Cz}$, and Pz) of the high-discard rate group (d) and the low-discard rate group (e) from both dominance (purple) and suppression (green) trials. Statistical significance associated with $t$ test is indicated by the asterisks of matching color $\left({ }^{*} p<0.05,{ }^{* *} p<0.01,{ }^{* * *} p<0.001\right)$. Error bars indicate $\pm 1 \mathrm{SE}$.

eight sets. This effectively eliminated physical stimulus differences associated with word length between the words sets. As described below, these eight sets of word pairs were then counterbalanced across presentation conditions and observers.

To induce interocular suppression, dynamic random texture patterns were created similar to those in Experiment 1. Each randomly generated texture pattern consisted of $40 \times 40$ cells and the size of the whole pattern was $4.7^{\circ} \times 4.7^{\circ}$. Cells of the texture patterns were randomly filled with red colors of two luminance levels with approximately equal frequency. This manipulation provided a better control across trials in terms of stimulus energy compared with the Mondrian frames used in Experiments 1 and 2 in which there was more variability in the local contrast across frames. The mean luminance level was approximately equal to the background (i.e., $47.5 \mathrm{~cd} / \mathrm{m}^{2}$ ). On each trial, three frames of the random texture patterns were presented immediately after one another for 100 ms each to create dynamically changing suppressive stimuli. The target word or letter string was presented for $100 \mathrm{~ms}$ with the second frame of the random texture patterns. All other aspects of stimuli were identical to those in Experiment 1.

Experiment 4 consisted of three sessions: a calibration session, the main experimental session, and a confirmation session. The goal of the calibration session was to determine multiple stimulus energy levels in preparation for the main experiment. The goal of the confirmation session was to measure the visibility levels resulting from the stimulus energy levels set during the calibration session and to assess any changes that occurred during the experimental session. This confirmation session was necessary because it was likely that increased discrimination performance would result from dark adaptation during the main experiment session (Purcell et al., 1983).

We used two tasks for the calibration and confirmation sessions: a lexical-decision task and a feature-discrimination task. The logic behind our use of the lexical-decision task was that it would indicate at what luminance level people could extract enough information from the word stimuli to know that some word, or word-like stimulus, was presented. The feature-discrimination task set a lower benchmark in determining at what luminance level the basic features of words (i.e., letter strings) could be extracted. In the lexical-decision task, observers had to determine whether a given letter string was a word or a nonword. Words were randomly drawn from the 768 different words used to create eight sets of semantically related pairs of words. Nonwords were created by randomly mixing alphabetical letters in which the frequency of each letter was drawn from the distribution of alphabetical letters in those 768 words. In addition, the letter-string length was drawn from the distribution of word length of the 768-word list. In the feature-discrimination task, observers had to determine whether a given stimulus was composed of homogeneous Os or Xs (e.g., XXXX vs OOOO). The length of this homogeneous letter string was randomly chosen from the same word length frequency distribution used to create nonwords described above. We assumed that this feature-discrimination task was easier at the same stimulus energy level compared with lexical-decision task because $\mathrm{X}$ is easily distinguishable from $\mathrm{O}$ due to the letters being composed of different simple features (Treisman and Gelade, 1980). In addition, even a single character or a fraction of a single character is as informative as to the whole letter string.

During the calibration session, we empirically derived four stimulus energy levels. First, we set the high stimulus energy level by adjusting the contrast of the random texture pattern and the letter string so that observers could reliably perform the lexical-decision task under interocular suppression. Because observers can perform a lexical-decision task with partially visible stimuli, the contrast of the random texture pattern was adjusted such that observers could recognize all the individual letters. This procedure was conducted such that observers had to report all char- 
a

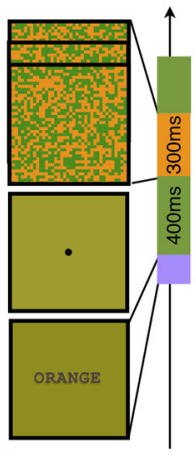

Left Eye
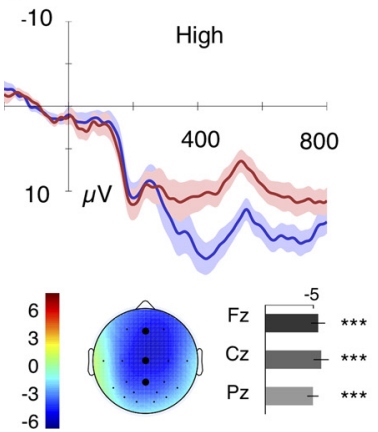

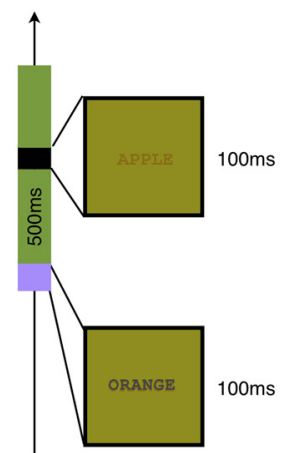

Right Eye d

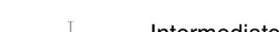

b

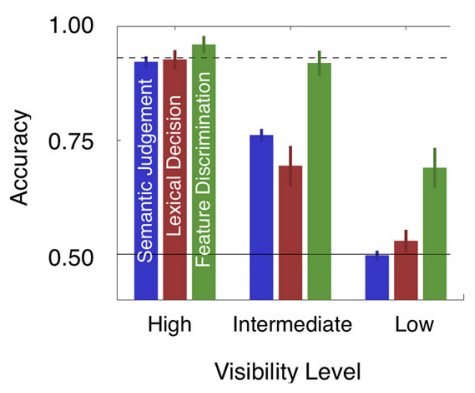

e

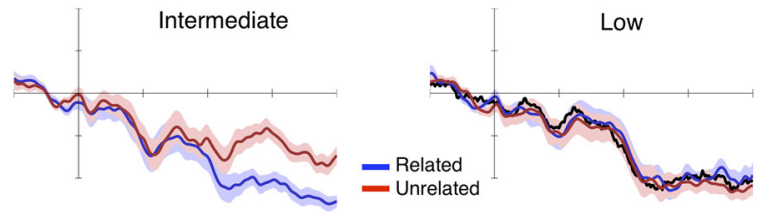

Figure 4. Stimuli and results of Experiment 4. a, Illustration of stimulus sequence of Experiment 4 (related pairs). Context word (ORANGE) is presented to the one eye for $100 \mathrm{~ms}$ and the first random texture frame is presented to the other eye after $400 \mathrm{~ms}$ of fixation. Three random texture frames are presented in sequence, one every $100 \mathrm{~ms}$. The target word (APPLE) is presented dichoptically for $100 \mathrm{~ms}$ with the second random texture frame. $\boldsymbol{b}$, Behavioral results of Experiment $4(N=16)$. Accuracy of the semantic-judgment task (blue), lexical-decision task (red), and feature-discrimination task (green) are plotted across the three visibility levels. Error bars indicate $\pm 1 \mathrm{SE}$. The chance level (50\%) is plotted with the black solid line and the accuracy associated with lexical-decision task performance without interocular suppression is plotted with the black dotted line. ERP waveforms and the N400 amplitude obtained from high (c), intermediate (d), and low-visibility (e) levels. Details are as in Figure $1, b$ and $c$. The black curve in e represents ERP waveform elicited by the monocular random texture pattern without target words.

random texture patterns without target words were presented to measure the neural response elicited by only the random texture patterns. All of these word-pair types and stimulus energy levels produced a total of 432 trials that were randomly interleaved. We counterbalanced the experimental factors across observers, resulting in 16 different combinations.

In the confirmation session, observers performed both the lexical-decision and the feature-detection tasks to determine the visibility levels accompanied with the four stimulus energy levels. In the lexical-decision task, 60 words and 60 nonwords were presented such that 15 words and 15 nonwords were assigned to each stimulus energy level. In the featuredetection task, 60 letter strings of Os and another 60 letter stings of Xs were generated and assigned to the four stimulus energy levels. Trials of the four stimulus energy levels were randomized and the order of the two tasks were counterbalanced across observers. We also measured the lexical-decision performance without interocular suppression at the lowest energy level using 15 words and 15 nonwords.

After all three sessions, we determined the high-, intermediate-, and low-visibility levels from the four word stimulus energy levels used. For the high-visibility level, we used the trials associated with the highest stimulus energy level (average contrast, 80\%; 33\% 94\%). For the low-visibility level, we used the trials associated with the lowest stimulus energy level obtained from the lexical-decision task without interocular suppression in which observer's accuracy was $\sim 90 \%$. This is because the featurediscrimination task performance tended to be above chance but the lexical-decision performance was close to chance at that level (average contrast, $15 \% ; 12-21 \%)$. For the intermediate-

acters of a given random letter string (length was $<4$ ) verbally and the experimenter confirmed its visibility across a sequence of 10 trials. The contrast of the random texture pattern set at this stage was used for all other tasks and sessions for that observer. Second, we set the lowest stimulus energy level in which accuracy of the lexical-decision task without interocular suppression was $\sim 90 \%$ correct ( 15 word and 15 nonword samples). Third and fourth, the stimulus energy levels that resulted in threshold-level lexical-decision task performance and threshold-level feature-discrimination task performance were obtained with a staircase procedure ( 2 up, 1 down) with dichoptic presentation of the random texture patterns (thresholds set to $\sim 75 \%$ correct for each task).

Figure $4 a$ illustrates the stimulus sequence of the semantic judgment task. A fixation point was presented dichoptically for $900 \mathrm{~ms}$. A context word was presented monocularly for $100 \mathrm{~ms}$ and a $400 \mathrm{~ms}$ blank period followed in which the fixation point was presented dichoptically. The random texture pattern was then presented for $300 \mathrm{~ms}$ to the eye opposite to the context word. The random texture changed its pattern every 100 $\mathrm{ms}$ while a target word was presented for $100 \mathrm{~ms}$ with the second random texture pattern. Observers judged whether the target word was related or unrelated to the context word using the gamepad. Related and unrelated responses were associated with two buttons using the left and the right index fingers (counterbalanced across observers).

In the main experimental session, observers performed the semantic judgment task with the four stimulus energy levels obtained during the calibration session. Note that we recorded electrophysiological responses only while observers were performing the semantic judgment task during the $\sim 3 \mathrm{~h}$ experimental session. From the eight sets of related word pairs, four sets were used to create related pairs for the four visibility levels and the other four sets were used to produce unrelated pairs, for a total of 384 trials. In addition, we also included 48 trials in which context words and visibility level, we selected a single energy level out of the two middle stimulus energy levels that produced near $75 \%$ correct performance of the semantic-judgment task for each subject (average contrast, 28\%; $16-$ $38 \%$ ). This complex procedure was necessary to tightly control the stimulus energy on an individual observer basis and to verify that the visibility of the stimuli was being measured precisely.

\section{Results}

\section{Experiment 1: Continuous flash suppression}

We first conducted a simple experiment to test whether semantic analysis occurs in the absence of awareness during interocular suppression. As shown in Figure $1 a$, we presented a clearly visible context word that established a semantic context and then presented a target word while a potent and dynamic stimulus was presented to the other eye. This type of interocular suppression, called continuous flash suppression (Tsuchiya and Koch, 2005), is particularly useful because it is easy to control the timing of suppression, allowing us to time lock the ERPs to the suppressed target words and determine whether the brain extracted their meaning even before a behavioral response was made.

We combinatorially created two semantic word-pair types (related and unrelated) and two trial types (suppression and dioptic control). During the suppression trials, a clearly visible context word was presented to both eyes and then a target stimulus was presented to one eye while Mondrian patches were presented to the other eye (Fig. 1a). On dioptic control trials, the target stimulus was embedded within the dominant Mondrian patches 
and was presented at the same location to the other eye, resulting in clearly visible target words. The purpose of the dioptic control trials was to interleave baseline events in which participants were aware of the targets and could, therefore, perform the task. This allowed us to verify that participants showed a normal N400 under viewing conditions in which the words were clearly visible. The critical question was whether we would observe an N400 on the suppression trials similar to the waveforms measured during the dioptic control trials.

Behavioral performance indicated that observers were not aware of the meaning of target words on suppression trials because accuracy was not different from the chance level $(50.2 \%$, $\left.t_{(5)}=0.2017, p>0.5\right)$. This was in sharp contrast to the accuracy of the semantic judgment on dioptic control trials $\left(82.4 \%, t_{(5)}=\right.$ $8.47, p<0.001){ }^{a}$

Figure $1, b$ and $c$, shows the ERPs averaged across six observers from electrode $\mathrm{Cz}$ and the $\mathrm{N} 400$ amplitudes from the dioptic control and suppression trials, respectively. During the dioptic control trials, the ERP waveforms elicited by the unrelated target words became more negative than the waveforms elicited by the related target words $\sim 300-400 \mathrm{~ms}$ after stimulus onset. Figure 1, $b$ and $c$, bottom, shows topographical distribution of the N400 amplitude along with the N400 amplitudes measured across the three key electrodes $(\mathrm{Fz}, \mathrm{Cz}$, and $\mathrm{Pz}$; marked with large dots on the topographic map) used in previous studies of the N400 (Kutas and Hillyard, 1980). These voltages were calculated by subtracting the ERPs elicited by unrelated target words from the ERPs elicited by the related target words within the temporal measurement window (i.e., 350-500 ms poststimulus), chosen based on previous $\mathrm{N} 400$ studies to minimize contamination from the P3 component (Vogel et al., 1998). The two-way repeatedmeasures ANOVA with trial type (dioptic control vs suppression) and channel $(\mathrm{Fz}, \mathrm{Cz}$, and $\mathrm{Pz})$ as factors showed only a significant main effect of trial type $\left(F_{(1,5)}=61.86, p<0.001\right)$. The $\mathrm{N} 400$ elicited by dioptically presented targets was significant across all three electrodes (Fig. 1b,c, asterisks). However, the N400 was completely absent on suppression trials.

\section{Experiment 2: Continuous flash suppression with a longer target word duration}

One might argue that the duration of the target presentation was too brief in Experiment 1 to expect any processing in the absence of awareness. For this reason, we increased the target presentation time and eliminated the interval between context word offset and the Mondrian frame onset (Fig. 2a). Performance of the semantic relatedness judgment was not significantly different from the chance level $\left(51.95 \%, t_{(7)}=1.17, p=0.28\right)$. This level of accuracy was in sharp contrast to the accuracy of the semantic judgment on dioptic-control trials $\left(91.97 \%, t_{(7)}=17.02\right.$, $p<0.001$ ).

Figure 2, $a$ and $c$, shows the averaged ERPs, across the eight observers, elicited by the target words from electrode $\mathrm{Cz}$ and the N400 amplitudes from the dioptic control and suppression trials, respectively. Because the contrast of the target words was gradually increased for $300 \mathrm{~ms}$, the averaged ERPs were time-locked to the time point $100 \mathrm{~ms}$ after the beginning of this contrast increasing phase. Similar to Experiment 1, the ERP waveforms elicited by the unrelated target words became more negative than the

${ }^{a}$ The performance in dioptic-control trials of Experiment 1 was poor (82.4\%) compared with dioptic-control trials of Experiment 2 (91.97\%) and high-visibility trials of Experiment 4 (92.4\%). Based on observers' spontaneous reports after the experiment, we think this relatively low level of performance is due to the difficulty of recognizing target words embedded within the Mondrian frames within a short period of time. waveforms elicited by the related target words during dioptic control trials $\sim 350-400 \mathrm{~ms}$, time-locked to the point of $33 \%$ contrast during the ramp up of the target word contrast. In comparison, the ERPs from the related and unrelated target words were similar during the suppression trials (Fig. $2 b$ ). The N400 component obtained with the temporal measurement window of 350-500 ms, and the two-way ANOVA with trial type (dioptic control vs suppression) and channel $(\mathrm{Fz}, \mathrm{Cz}$, and $\mathrm{Pz}$ ) as factors showed only a significant main effect of trial type $\left(F_{(1,7)}=0.12 .69\right.$, $p<0.01$ ), replicating Experiment 1 even with the long target word presentation time.

Our results show that semantic analysis of word stimuli did not occur in the absence of awareness when interocular suppression was induced by continuous flashes of multiple Mondrian stimuli. However, one might argue that continuous flash suppression attenuates the strength of the suppressed stimulus so drastically that its residual effectiveness is nil. We know, after all, that continuous flash suppression is much more robust than conventional binocular rivalry. With conventional binocular rivalry, perception alternates spontaneously every few seconds between the two dissimilar monocular stimuli, whereas with continuous flash suppression, perceptual dominance of one monocular stimulus (the Mondrian) may endure for half a minute or longer before the suppressed stimulus achieves dominance. Moreover, probe-detection tasks have shown that threshold elevations during suppression phases induced by continuous flash suppression are approximately three times larger than threshold elevations during suppression phases of binocular rivalry (Tsuchiya and Koch, 2005; Tsuchiya et al., 2006). So it is reasonable to question whether the absence of a neural signature of semantic analysis outside of awareness in Experiments 1 and 2 might be attributable to the potency of continuous flash suppression. This possibility led us to measure the N400 component elicited by words presented outside of awareness using binocular rivalry to induce interocular suppression.

\section{Experiment 3: Binocular rivalry}

Testing the hypothesis that semantic analysis is performed during binocular rivalry, unlike continuous-flash suppression, required us to make several changes to our experimental procedures. This was necessary because perception changes unpredictably during binocular rivalry and we needed methods to ensure that we were presenting a stimulus to an eye that was either dominant or suppressed. First, we used a vertical and a horizontal grating as the rivaling stimuli and each context word was embedded within the central part of the horizontal grating (Fig. 3a). Observers triggered the presentation of a context word such that they waited until the vertical pattern became dominant (i.e., for suppression trials) or until the horizontal pattern became dominant (i.e., for dominance trials), and then pressed a designated button to trigger the brief presentation of the context word. The order of dominance and suppression trials was randomized and the trial type was cued before each trial. Second, instead of presenting a competing stimulus that interfered with the visibility of target words (as in Experiments 1 and 2), the visibility of the context words was challenged by rivalry suppression and target words were clearly presented to both eyes at the end of the trial. This change is critical for controlling the interval between the context and target words presentations. If a clearly visible context word was presented, then the interval between the context and target word could vary between trial types because the onset and duration of dominance phases are unpredictable on each trial during binocular rivalry. Third, to reduce perceptual reversals induced by visual transients 
(Walker and Powell, 1979; Blake et al., 1990), target words were gradually introduced by increasing and then decreasing their contrast. Finally, despite these countermeasures, we anticipated that on a subset of trials observers might actually see the context word due to dominance reversals, so we also provided another button with which observers could indicate a trial when they clearly saw the context word. Note that the procedure described above is similar to the procedure used by Zimba and Blake (1983).

Behavioral performance of the semantic judgment task on dominance trials was highly accurate $\left(90.3 \%, t_{(7)}=26.68, p<\right.$ $0.001)$. In contrast, the observers' responses on suppressed trials were not different from chance $\left(51.0 \%, t_{(7)}=0.51, p>0.5\right)$.

Figure $3, b$ and $c$, shows the ERPs averaged across eight observers from electrode $\mathrm{Cz}$ and the N400 amplitudes from dominance and suppression trials, respectively. Similar to our findings in Experiment 1, the ERPs elicited by unrelated relative to related target words on dominance trials produced an N400 beginning $\sim 300-400 \mathrm{~ms}$ after the onset of target word. Consistent with this observation, N400 amplitudes (Fig. 3b,c, bottom) obtained from three key electrodes $(\mathrm{Fz}, \mathrm{Cz}$, and $\mathrm{Pz}$ ) were significantly modulated by the semantic relatedness of the target word to the clearly presented context word. However, no significant N400 was elicited on suppressed trials. That is, the waveforms across all three key electrodes were not significantly modulated by the semantic relatedness of the context and target words, mirroring performance of the semantic judgment task on these trials. As with Experiments 1 and 2, the two-way ANOVA revealed a significant difference between dominance and suppression trials $\left(F_{(1,7)}=8.46\right.$, $p<0.05)$.

An additional facet of the findings from Experiment 3 was revealed when we examined the behavioral and ERP data from individual observers. Even though no statistically significant N400 was observed in the grand average waveforms during the suppression trials, the ERP waveforms elicited by unrelated target words on these trials were numerically more negative than those elicited by related target words (Fig. 3c). For a subset of the observers, the N400 amplitudes during suppression trials were comparable to those elicited during the dominance trials. This suggests that for some of our observers the context words on suppression trials may have been at least partially visible on a proportion of trials. Support for this hypothesis came from an analysis in which we sorted the participants based on their rates of discarding trials due to incomplete suppression and examined the N400s elicited.

We asked observers to report when they could recognize the context word presented during the suppression phase of rivalry and to discard these trials. We reasoned that observers who became aware of context words due to weak or incomplete suppression would have discarded more trials. To maintain sufficient statistical power to examine the relationship between discard rate and N400 effects, we split the observers into two groups based on the median of the discard rate: a high-discard group and a lowdiscard group. The discard rates of these two groups were significantly different $\left[t_{(6)}=4.60, p<0.01\right.$; mean discard: $7 \pm 3.8 \%$ $($ mean $\pm \mathrm{SD}$ ) for the low-discard group, $18 \pm 3.8 \%$ for the highdiscard group] even though the semantic judgment performance was similar between these two groups. Mean accuracy on dominance trials was $90.5 \pm 4.4 \%$ and $90 \pm 4.8 \%$ for the high- and low-discard rate groups and the mean accuracy on the suppression trials was $49 \pm 6.4 \%$ and $53 \pm 4.2 \%$ for the high- and the low-discard group, respectively. A two-way mixed-design ANOVA with trial type (dominance vs suppression) and group (high vs low) resulted in no difference in accuracy between the high and low groups $\left(F_{(1,6)}=0.52, p=0.50\right)$.

With this chance-level semantic judgment performance in the suppression trials in mind, we looked at the amplitude of the N400 in these two groups. Figure 3 shows that N400 amplitudes obtained from the high-discard group (Fig. $3 d$ ) and the lowdiscard group (Fig. 3e) for both the dominance and the suppression trials. A two-way mixed-model ANOVA revealed that there is a significant interaction between group (high vs low discard rate) and trial type (dominance vs suppression) $\left(F_{(1,6)}=12.80\right.$, $p<0.05)$. Consistent with this statistical observation, N400 amplitudes during all dominance and suppression trials for the highdiscard rate group are significantly different from zero, whereas the N400 amplitudes are only significantly different from zero during dominance trials of the low-discard rate group (Fig. 3, asterisks).

In one way, these findings suggest that observers may have adopted a higher criterion for the semantic-relatedness judgment on suppression trials than dominance trials, particularly participants in the high-discard group. This is consistent with the idea that our observers had to perform the additional task of reporting when they became aware of the context words on suppression trials and these dual-task demands could have led to the adoption of a higher criterion than on the single-task trials of the dominance condition, resulting in chance-level semantic judgment performance. Thus, when we categorized the data from the observers based on their behavioral report of the words breaking suppression, and their becoming aware of the word, it appears that words that were incompletely suppressed (i.e., partially visible) did elicit an N400 showing that they were semantically processed. Alternatively, the observers in the high-discard group may have processed the context words during suppression with greater efficiency than did observers in the low-discard group (e.g., due to faster reading abilities); this could also explain why high-discard observers also reported a greater proportion of perceived trials. Essentially, for the high-discard rate observers, the stimulus and timing parameters of the experiment were closer to the optimum values for placing the stimuli at threshold for a significant N400 to be observed in the absence of awareness.

These competing explanations motivated Experiment 4, where we explicitly examined whether different levels of visibility (i.e., stimulus parameters) could elicit different degrees of semantic analysis. In Experiment 4, we directly addressed the contributions of stimulus visibility and subjective criterion levels by parametrically manipulating the visibility of stimuli during interocular suppression within each observer. This more precise quantification would then tell us whether the N400 is truly absent when people are unaware of the suppressed stimuli, as Experiments 1,2 , and 3 suggest.

\section{Experiment 4: Interocular suppression across multiple visibility levels}

The notion of stimulus visibility has been a central issue when determining whether semantic analysis occurs in the absence of awareness. Over the past 30 years, the subliminal priming procedure has contributed a large body of empirical evidence following the seminal work of Marcel (1983). In this procedure, observers respond to clearly visible target words followed by masked prime words. To insure that observers are not aware of the masked prime words, prime visibility has been measured separately with observers performing several types of discrimination and detection tasks involving the masked prime words themselves. Several 
studies report that even when discrimination of the masked primes is at chance, observers' responses to the target words are faster when the target words are semantically related to the prime words (Greenwald et al., 1996; Dehaene et al., 1998; Kunde et al., 2003). However, this literature underscores that, from a methodological perspective, determining whether or not semantic analysis occurs in the complete absence of awareness is a nontrivial matter (Adams, 1957; Eriksen, 1960; Merikle and Reingold, 1998). Specifically, several studies found that facilitated responses to target words were correlated with the prime words' visibility using rigorous measures of whether the primes were seen (Nolan and Caramaz, 1982; Dark, 1988; Kouider and Dehaene, 2007). In addition, the procedure of having participants discriminate the masked prime words has been criticized because the difficulty of discriminating items near threshold may promote higher rates of guessing when observers give up on the task (Pratte and Rouder, 2009).

With these concerns in mind, in Experiment 4 we directly varied the visibility of the suppressed stimulus across multiple levels to probe for the presence of semantic processing in the absence of awareness. This allowed us to directly manipulate stimulus visibility and assess contributions of decision criteria in a controlled manner. Our manipulation also allowed us to rule out the possibility that semantic analysis of interocularly suppressed stimuli occurs, but so far we have only tested for its presence in situations where the effective stimulus strength is too weak to possibly support such processing.

We manipulated the stimulus visibility parametrically by varying the stimulus energy of the words relative to the suppressing stimuli and, thus, suppression strength within a stimulus sequence similar to Experiment 1 (Fig. 4a). Specifically, we set three levels of visibility individually for each observer by manipulating the luminance of the words and the suppressing stimuli. At one extreme, we set a high-visibility level titrated such that observers could reliably recognize the dichoptically presented word stimuli (i.e., with $90-95 \%$ accuracy). Based on the previous two experiments, we expected to observe a robust $\mathrm{N} 400$ at the high-visibility level. At the other extreme, a low-visibility level was set such that observers could not determine the meaning of the word stimuli but could extract some of the basic features of stimuli (i.e., some fragment of the letters in the words). We did this because stringent experimental conditions in which even simple features cannot be extracted may preclude any processing in the absence of awareness (Gaillard et al., 2006). If the N400 is obtained at this low-visibility level, as in high-discard group of Experiment 3, it would provide a lower boundary where semantic processing occurs in the absence of awareness without being attributable to differences in subjective decision criteria, as was possible in the task used in Experiment 3. In between the extreme levels of visibility, we set an intermediate level such that observers could extract the meaning of word stimuli significantly above the chance level.

Previous research has indicated that this intermediatevisibility level is critical. Previously, Luck and colleagues (Luck et al., 1996; Vogel et al., 1998) measured the N400 amplitude while disrupting the processing of target words during attentional blink and masking by simultaneous visual noise so that behavioral accuracies of these two distinct manipulations produced essentially the same intermediate levels of discrimination accuracy (i.e., $\sim 60 \%$, with $50 \%$ being chance). The N400 elicited by target words presented during the attentional blink was comparable to the N400 elicited by targets presented during baseline trials that resulted in near ceiling levels of discrimination accuracy. In con- trast, the N400 elicited by target words presented simultaneously with visual masking noise was attenuated compared with the response to the baseline target words. This previous work demonstrates that some manipulations, such as simultaneous masking, that result in moderate disruptions of awareness of target words are due to interference with perceptual processing that disrupts the N400, whereas manipulations of awareness that operate after semantic analysis, such as processing during the attentional blink, result in a perfectly normal N400. By including this intermediate-visibility level, we sought to determine whether moderate impairments in discrimination accuracy would be accompanied by attenuation of the N400 or a normal N400.

We predicted that if semantic processing consistently occurs at this intermediate-visibility level, the amplitude of the N400 should be comparable to when the stimuli are clearly visible (i.e., the high-visibility level). This prediction is also consistent with several priming studies (Abrams and Greenwald, 2000; Costello et al., 2009). In contrast, if interocular suppression operates to interfere with the processing of visual input and, thereby, provides the degraded visual input to the brain areas that perform semantic analysis, then we predicted that the averaged $\mathrm{N} 400$ would be attenuated at the intermediatevisibility level relative to the words presented at the highvisibility level, similar to when physically degraded stimuli are provided (Vogel et al., 1998).

Figure $4 b$ shows the behavioral performance obtained from the feature-discrimination (green), lexical-decision (red), and semantic judgment (blue) tasks across the three visibility levels (as described in Materials and Methods, above). The lexical-decision task performance without interocular suppression at the lowest stimulus energy level (Fig. $4 b$, dotted black line) and the chance level performance (50\%; Fig. $4 b$, solid black line) are plotted. A repeated-measures, two-way ANOVA with factors of task (feature discrimination, lexical decision, or semantic judgment) and visibility level (high, intermediate, or low) verified that accuracy in the three tasks decreased significantly with decreasing visibility level $\left(F_{(2,30)}=116.11, p<0.001\right)$. In particular, performance at the low-visibility levels demonstrates the effectiveness of our stimulus manipulation. That is, chance-level semantic judgment performance and above chance-level feature discrimination performance indicate that interocular suppression was well established but not so potent as to abolish all traces of the representation of the suppressed words.

Figure $4, c, d$, and $e$, show the averaged ERPs from electrode $\mathrm{Cz}$ for the three visibility levels and the N400 amplitudes. Figure $4 e$ shows the ERPs elicited by the monocular random texture pattern alone (black curve). At the high-visibility level, the semantically unrelated target words elicited a more negative-going potential between 350 and 450 ms poststimulus compared with the waveforms elicited by semantically related target words. This N400 reflects the neural response during conscious semantic analysis (as verified by the extensive behavioral calibration and testing; Fig. $4 b$ ). In contrast, Figure $4 d$ shows that the amplitude of N400 component was attenuated at the intermediate-visibility level. At the low-visibility level, the N400 was again absent such that both the related and unrelated words produced ERP responses that were indistinguishable. A two-way ANOVA with the factors of electrode $(\mathrm{Pz}, \mathrm{Cz}$, and $\mathrm{Fz})$ and visibility level (high, intermediate, and low) confirmed that there was a significant effect of the visibility level on the amplitude of the voltage difference between related and unrelated words $\left(F_{(2,30)}=\right.$ 
29.16, $p<0.001){ }^{b}$ Next, we ran a three-way ANOVA with only the two critical visibility levels (high and intermediate) and the factors of electrode $(\mathrm{Pz}, \mathrm{Cz}$, and $\mathrm{Fz}$ ) and trial type (related and unrelated). This resulted in a significant effect of visibility level $\left(F_{(1,15)}=46.60, p<0.001\right)$, electrode site $\left(F_{(2,30)}=8.17, p<\right.$ $0.01)$, and trial type $\left(F_{(1,15)}=76.80, p<0.001\right)$. The latter two main effects verify that the N400 we observed differing between the high- and intermediate-visibility levels had the defining features of sensitivity to violations of semantic context and a distribution with a frontocentral maximum. This significant effect of visibility level between high and intermediate conditions confirms the predictions of an account in which the brain areas processing semantic information receive degraded representations from the suppressed eye.

This attenuation in both the ERP response and behavioral performance is similar to the attenuation of the N400 obtained when the target stimuli are physically degraded with simultaneous masking noise (Vogel et al., 1998). More importantly, given that observers could see some features at the low-visibility level (i.e., $\mathrm{X}$ or O), the absence of the N400 at that visibility level strongly indicates that semantic analysis does not occur in the absence of awareness of the meaning of a stimulus when induced by interocular suppression.

\section{Discussion}

The findings of all four experiments converge in demonstrating that no N400 is observed when a critical word is completely erased from awareness by interocular suppression. This was despite the fact that we measured the N400 during two types of interocular suppression. It is well established that the N400 is the process-specific electrophysiological index of semantic analysis of words, pictures, sounds, and smells (Kutas and Federmeier, 2000, 2011). Our findings definitively show that stimuli completely suppressed from awareness do not engage those processes involved in semantic analysis that are tapped by the N400. We cannot, of course, rule out the possibility that there exists neural activity in the brain related to semantic analysis that fails to register using noninvasive electrophysiological measurements.

\section{Stimulus visibility matters}

Our parametric manipulation of stimulus energy produced different levels of visibility and, thus, different degrees of awareness during interocular suppression. The pattern of N400 results we obtained allows us to draw strong conclusions about the nature of semantic processing during such suppression. Specifically, we found that the N400 was attenuated with increasing magnitude of suppression and became absent when performance of the semantic relatedness judgment approached chance (i.e., 50\%) despite the fact that simple feature information could be extracted. These findings are similar to the patterns of N400 activity obtained when target words are physically degraded by superimposed masking visual noise (Vogel et al., 1998). Thus, our findings across Experiments 1, 2, 3, and 4 provide converging evidence that semantic analysis does not occur in the absence of awareness during interocular suppression.

This conclusion is striking because previous research has found that the N400 is sensitive enough to measure semantic

\footnotetext{
${ }^{b}$ It is fairly well known among ERP researchers that there are individual differences in the morphology of ERP components (Luck, 2005; Woodman, 2010). However, the effect of our visibility manipulation on the N400 (semantically related vs unrelated) was strikingly consistent across observers. To demonstrate the robustness of this pattern of effects at the individual-subject level, we have made these data available for download in the Supplemental material for the interested reader.
}

processing of information during other paradigms that impair awareness, such as the attentional blink (Luck et al., 1996; Giesbrecht et al., 2007). Below, we describe how the integrative framework of the global neuronal workspace theory reconciles these results with previous findings that seemingly show semantic analysis of stimuli subjected to interocular suppression.

\section{Global neuronal workspace theory}

The biological basis of consciousness has been studied extensively by neuroscientists and a number of models have been proposed (for review, see Edelman et al., 2011). Among these models, the dichotomies of phenomenal and access consciousness (Block, 2005) and conscious and unconscious processing (Cheesman and Merikle, 1986; Greenwald et al., 1996) have been criticized on theoretical and methodological grounds (Holender and Duscherer, 2004; Kouider and Dehaene, 2007; Kouider et al., 2010). Instead, we believe that the global neuronal workspace theory provides a useful framework for explaining a large body of empirical evidence obtained from visual masking, attention paradigms, and now interocular suppression.

According to the global neuronal workspace theory, sensory information is consciously available for high-level processing when bidirectional, self-sustained activation loops are established between the sensory and workspace neurons distributed over several brain areas, including prefrontal and parietal cortices (Dehaene et al., 2001, 2003). Under this framework, Dehaene and colleagues (Dehaene et al., 2006; Kouider and Dehaene, 2007) distinguished two types of nonconscious states: the subliminal and the preconscious states. In the subliminal state, sensory information is too weak to reach the workspace; in the preconscious state, the sensory information is fed into the workspace but it does not receive top-down amplification.

The preconscious and subliminal distinction accounts for empirical differences between states of awareness using visual masking and attentional paradigms in previous studies (Dehaene et al., 2006). The global neuronal workspace theory proposes that stimulus visibility (i.e., stimulus energy) determines whether a given stimulus is processed under the subliminal and preconscious state. Sergent and Dehaene (2004) asked observers to rate the visibility of a target stimulus during visual-masking and attentionalblink paradigms. In an attentional-blink paradigm, they found that subjective ratings produced a discrete bimodal distribution while the temporal proximity of the two targets was varied (see also Vul et al., 2009). This means that observers either could recognize the targets with confidence or could not recognize the targets at all. In contrast, in a visual-masking paradigm, the subjective ratings spread out when the temporal proximity of the target and the mask was varied, resulting in a unimodal distribution of rating across the visibility scale. In other words, the visual representation of a target is degraded with increasing strength of visual masking.

Consistent with the stimulus level-dependent difference between visual-masking and attentional-blink paradigms, the presence or absence of the N400 indicates that semantic analysis in the absence of awareness occurs during the attentional-blink but not masking paradigms. In attention-blink studies, comparable N400s were elicited by target words that were missed during the attentional blink and target words were consciously available (Luck et al., 1996; Giesbrecht et al., 2007). In contrast, Brown and Hagoort (1993) found no N400 when the discrimination of masked words was near chance during a subliminal priming procedure (see also Reiss and Hoffman, 2006, but see Deacon et al., 2000; Kiefer, 2002). This implies that stimuli are registered and analyzed within the workspace during the attentional blink, but 
in visual masking, feedforward activity produced by sensory stimulation progressively dies out and fails to be registered within the higher-level workspace and, thus, precludes semantic processing.

How, then, can we understand the processing characteristics of invisible stimuli due to interocular suppression? In the context of global neuronal workspace theory, interocular suppression is similar to visual masking in the nature of processing, which denies a stimulus access to awareness. This is because stimulus visibility gradually changes with stimulus energy level in interocular suppression, as revealed by the clear difference between performance using the feature discrimination, semantic-relatedness judgment, and lexical-decision tasks at the intermediate visibility level. Stimulus processing, in other words, is sensitive to the stimulus energy level even though that stimulus does not compete for the attention of the observer. Note, however, that in comparing masking and interocular suppression, we do not intend to imply that the two phenomena arise from common neural processes. Indeed, there are reasons to believe that is not the case (Tse et al., 2005; Breitmeyer et al., 2008) at least when it comes to backward masking (but for a discussion of the equivalence of interocular suppression and dichoptic, simultaneous masking, see Baker and Graf, 2009).

\section{When a partially visible stimulus is informative}

How then can we explain previous results showing high-level processing in the absence of awareness during interocular suppression? Kouider and colleagues (Kouider and Dupoux, 2004; Kouider and Dehaene, 2007; Kouider et al., 2010) proposed the partial-awareness hypothesis by advancing the tripartite distinction of the global neuronal workspace theory. This hypothesis states that representations are hierarchically organized (from low-level features to high-level representations of meaning) and observers can access these different levels of representation independently. Specifically, they proposed that partial awareness is due to the brain combining representations across levels while being aware of information at low levels and unaware of the representations at higher levels (for a related proposal, see Di Lollo et al., 2000).

This partial-awareness hypothesis can explain a large body of empirical results from the subliminal priming procedure and anomalous findings claiming semantic processing in the absence of awareness (Kouider and Dupoux, 2004; Pratte and Rouder, 2009). In particular, Kouider and Dupoux (2004) found that faster reaction times in the masked-priming procedure were associated with chance-level performance in a lexical-decision task, but above chance performance in a feature-discrimination task. Considering that a small set of prime and target stimuli are often repeatedly used and, thus, a partially visible stimulus is informative (Lupker, 1986), Kouider and Dupoux (2004) argued that consciously available partial information produced an illusion of subliminal semantic processing.

Similar illusions of subliminal semantic processing appear to account for findings from previous interocular suppression studies. These studies also used a small set of stimuli that were presented repeatedly. This means that discrimination performance being at chance may not have been a sensitive enough objective measure of awareness because the ability to discriminate a couple of features may have been sufficient to infer the meaning (Williams et al., 2004; Fang and He, 2005; Jiang et al., 2006; Almeida et al., 2008). This concern can also be applied to the measure of suppression duration as a proxy for awareness if the same stimuli are used more than once, because observers may have used par- tially available information to infer the object that was presented (Jiang and He, 2006; Yang et al., 2007). Considering, however, that all these studies used images of objects rather than words, we cannot rule out the possibility that object meaning is registered during suppression.

\section{Notes}

Supplemental material for this article is available at http://www. psy.vanderbilt.edu/faculty/woodman/kangetalJoN2011/WordPairs Experiment4.pdf. This material provides word pair sets for Experiment 4 for download and inspection. This material has not been peer reviewed.

\section{References}

Abrams RL, Greenwald AG (2000) Parts outweigh the whole (word) in unconscious analysis of meaning. Psychol Sci 11:118-124.

Adams JK (1957) Laboratory studies of behavior without awareness. Psychol Bull 54:383-405.

Almeida J, Mahon BZ, Nakayama K, Caramazza A (2008) Unconscious processing dissociates along categorical lines. Proc Nat Acad Sci U S A 105:15214-15218.

Baker DH, Graf EW (2009) On the relation between dichoptic masking and binocular rivalry. Vision Res 49:451-459.

Besner D, Smith MC, MacLeod CM (1990) Visual word recognition: a dissociation of lexical and semantic processing. J Exp Psychol Learn Mem Cogn 16:862-869.

Blake R (1988) Dichoptic reading: the role of meaning in binocular rivalry. Percept Psychophys 44:133-141.

Blake R, Westendorf D, Fox R (1990) Temporal perturbations of binocular rivalry. Percept Psychophys 48:593-602.

Block N (2005) Two neural correlates of consciousness. Trends Cogn Sci 9:46-52.

Brainard DH (1997) The psychophysics toolbox. Spat Vis 10:433-436.

Breitmeyer BG, Koç A, Oğmen H, Ziegler R (2008) Functional hierarchies of nonconscious visual processing. Vision Res 48:1509-1513.

Brown C, Hagoort P (1993) The processing nature of the N400: evidence from masked priming. J Cogn Neurosci 5:34-44.

Brown M, Stolz JA, Besner D (2006) Dissociative effects of stimulus quality on semantic and morphological contexts in visual word recognition. Can J Exp Psychol 60:190-199.

Cave CB, Blake R, McNamara TP (1998) Binocular rivalry disrupts visual priming. Psychol Sci 9:299-302.

Cheesman J, Merikle PM (1986) Distinguishing conscious from unconscious perceptual processes. Can J Psychol 40:343-367.

Costello P, Jiang Y, Baartman B, McGlennen K, He S (2009) Semantic and subword priming during binocular suppression. Conscious Cogn $18: 375-382$

Dark VJ (1988) Semantic priming, prime reportability, and retroactive priming are interdependent. Mem Cognit 16:299-308.

Deacon D, Hewitt S, Yang C, Nagata M (2000) Event-related potential indices of semantic priming using masked and unmasked words: evidence that the N400 does not reflect a post-lexical process. Brain Res Cogn Brain Res 9:137-146.

Dehaene S, Naccache L, Le Clec'H G, Koechlin E, Mueller M, DehaeneLambertz G, van de Moortele PF, Le Bihan D (1998) Imaging unconscious semantic priming. Nature 395:597-600.

Dehaene S, Naccache L, Cohen L, Bihan DL, Mangin JF, Poline JB, Rivière D (2001) Cerebral mechanisms of word masking and unconscious repetition priming. Nat Neurosci 4:752-758.

Dehaene S, Sergent C, Changeux JP (2003) A neuronal network model linking subjective reports and objective physiological data during conscious perception. Proc Nat Acad Sci U S A 100:8520-8525.

Dehaene S, Changeux JP, Naccache L, Sackur J, Sergent C (2006) Conscious, preconscious, and subliminal processing: a testable taxonomy. Trends Cogn Sci 10:204-211.

Di Lollo V, Enns JT, Rensink RA (2000) Competition for consciousness among visual events: the psychophyscis of reentrant visual processes. J Exp Psychol Gen 129:481-507.

Edelman, GE, Gally JA, Baars BJ (2011) Biology of consciousness. Front Psychol 2:4.

Eriksen CW (1960) Discrimination and learning without awareness: a methodological survey and evaluation. Psychol Rev 67:279-300. 
Fang F, He S (2005) Cortical responses to invisible objects in the human dorsal and ventral pathways. Nat Neurosci 8:1380-1385.

Gaillard R, Del Cul A, Naccache L, Vinckier F, Cohen L, Dehaene S (2006) Nonconscious semantic processing of emotional words modulates conscious access. Proc Nat Acad Sci U S A 103:7524-7529.

Giesbrecht B, Sy JL, Elliott JC (2007) Electrophysiological evidence for both perceptual and postperceptual selection during the attentional blink. J Cogn Neurosci 19:2005-2018.

Greenwald AG, Draine SC, Abrams RL (1996) Three cognitive markers of unconscious semantic activation. Science 273:1699-1702.

Haynes JD, Rees G (2005) Predicting the stream of consciousness from activity in human visual cortex. Curr Biol 15:1301-1307.

Holender D, Duscherer K (2004) Unconscious perception: the need for a paradigm shift. Percept Psychophys 66:872-881.

Jiang Y, He S (2006) Cortical responses to invisible faces: dissociating subsystems for facial-information processing. Curr Biol 16:2023-2029.

Jiang Y, Costello P, Fang F, Huang M, He S (2006) A gender- and sexual orientation-dependent spatial attentional effect of invisible images. Proc Nat Acad Sci U S A 103:17048-17052.

Kiefer M (2002) The N400 is modulated by unconsciously perceived masked words: further evidence for an automatic spreading activation account of N400 priming effects. Brain Res Cogn Brain Res 13:27-39.

Kim CY, Blake R (2005) Psychophysical magic: rendering the visible invisible.' Trends Cogn Sci 9:381-388.

Kouider S, Dehaene S (2007) Levels of processing during non-conscious perception: a critical review of visual masking. Philos Trans R Soc Lond B Biol Sci 362:857-875.

Kouider S, Dupoux E (2004) Partial awareness creates the "illusion" of subliminal semantic priming. Psychol Sci 15:75-81.

Kouider S, de Gardelle V, Sackur J, Dupoux E (2010) How rich is consciousness? The partial awareness hypothesis. Trends Cogn Sci 14:301-307.

Kunde W, Kiesel A, Hoffmann J (2003) Conscious control over the content of unconscious cognition. Cognition 88:223-242.

Kutas M, Federmeier KD (2000) Electrophysiology reveals semantic memory use in language comprehension. Trends Cogn Sci 4:463-470.

Kutas M, Federmeier KD (2011) Thirty years and counting: finding meaning in the N400 component of the event related brain potential (ERP). Annu Rev Psychol 62:621-647.

Kutas M, Hillyard SA (1980) Reading senseless sentences: brain potentials reflect semantic incongruity. Science 207:203-205.

Lau EF, Phillips C, Poeppel D (2008) A cortical network for semantics: (de)constructing the N400. Nat Rev Neurosci 9:920-933.

Lee SH, Blake R, Heeger DJ (2007) Hierarchy of cortical responses underlying binocular rivalry. Nat Neurosci 10:1048-1054.

Lin Z, He S (2009) Seeing the invisible: the scope and limits of unconscious processing in binocular rivalry. Prog Neurobiol 87:195-211.

Luck SJ (2005) An introduction to the event-related potential technique. Cambridge, MA: MIT.

Luck SJ, Vogel EK, Shapiro KL (1996) Word meanings can be accessed but not reported during the attentional blink. Nature 383:616-618.

Lupker (1986) Conscious identification: where do you draw the line? Behav Brain Sci 9:37-38.

Marcel AJ (1983) Conscious and unconscious perception: experiments on visual masking and word recognition. Cogn Psychol 15:197-237.

Merikle PM, Reingold EM (1998) On demonstrating unconscious perception: comment on Draine and Greenwald (1998). J Exp Psychol Gen 127:304-310.
Moradi F, Koch C, Shimojo S (2005) Face adaptation depends on seeing the face. Neuron 45:169-175.

Nolan KA, Caramazza A (1982) Unconscious perception of meaning: a failure to replicate. Bull Psychon Soc 20:23-26.

Nunez PL, Srinivasan R (2006) Electric fields of the brain: the neurophysics of EEG, 2nd Edition. Oxford: Oxford UP.

O'Seaghdha PG, Marin JW (1997) Mediated semantic-phonological priming: Calling distant relatives. J Mem Lang 36:226-252.

Pelli DG (1997) The VideoToolbox software for visual psychophysics: transforming numbers into movies. Spat Vis 10:437-442.

Pratte MS, Rouder JN (2009) A task-difficulty artifact in subliminal priming. Atten Percept Psychophys 71:1276-1283.

Purcell DG, Stewart AL, Stanovich KE (1983) Another look at semantic priming without awareness. Percept Psychophys 34:65-71.

Reiss JE, Hoffman JE (2006) Object substitution masking interferes with semantic processing: evidence from event-related potentials. Psychol Sci 17:1015-1020.

Sergent C, Dehaene S (2004) Is consciousness a gradual phenomenon? Evidence for an all-or-none bifurcation during the attentional blink. Psychol Sci 15:720-728.

Sterzer P, Haynes JD, Rees G (2008) Fine-scale activity patterns in high-level visual areas encode the category of invisible objects. J Vis 8:10.1-10.12.

Tong F, Meng M, Blake R (2006) Neural bases of binocular rivalry. Trends Cogn Sci 10:502-511.

Treisman AM, Gelade G (1980) A feature-integration theory of attention. Cogn Psychol 12:97-136.

Tse PU, Martinez-Conde S, Schlegel AA, Macknik SL (2005) Visibility, visual awareness, and visual masking of simple unattended targets are confined to areas in the occipital cortex beyond human V1/V2. Proc Nat Acad Sci U S A 102:17178-17183.

Tsuchiya N, Koch C (2005) Continuous flash suppression reduces negative afterimages. Nat Neurosci 8:1096-1101.

Tsuchiya N, Koch C, Gilroy LA, Blake R (2006) Depth of interocular suppression associated with continuous flash suppression, flash suppression, and binocular rivalry. J Vis 6:1068-1078.

Vogel EK, Luck SJ, Shapiro KL (1998) Electrophysiological evidence for a postperceptual locus of suppression during the attentional blink. J Exp Psych Hum Percept Perform 24:1656-1674.

Vogel EK, Woodman GF, Luck SJ (2005) Pushing around the locus of selection: evidence for the flexible-selection hypothesis. J Cogn Neurosci 17:1907-1922.

Vul E, Hanus D, Kanwisher N (2009) Attention as inference: selection is probabilistic; responses are all-or-none samples. J Exp Psychol Gen 138:546-560.

Walker P, Powell DJ (1979) The sensitivity of binocular rivalry to changes in the nondominant stimulus. Vision Res 19:247-249.

Williams MA, Morris AP, McGlone F, Abbott DF, Mattingley JB (2004) Amygdala responses to fearful and happy facial expressions under conditions of binocular suppression. J Neurosci 24:2898-2904.

Woodman GF (2010) A brief introduction to the use of event-related potentials (ERPs) in studies of perception and attention. Atten Percept Psychophys 72:2031-2046.

Wunderlich K, Schneider KA, Kastner S (2005) Neural correlates of binocular rivalry in the human lateral geniculate nucleus. Nat Neurosci 8:1595-1602.

Yang E, Zald DH, Blake R (2007) Fearful expressions gain preferential access to awareness during continuous flash suppression. Emotion 7:882-886.

Zimba LD, Blake R (1983) Binocular rivalry and semantic processing: out of sight, out of mind. J Exp Psych Hum Percept Perform 9:807-815. 